\title{
ARTICLE Fisetin alleviates sepsis-induced multiple organ dysfunction in mice via inhibiting p38 MAPK/MK2 signaling
}

\author{
Hai-feng Zhang ${ }^{1}$, Hai-bo Zhang ${ }^{2}$, Xue-ping Wu' ${ }^{1}$, Ya-ling Guo ${ }^{1}$, Wei-dong Cheng ${ }^{1}$ and Feng Qian ${ }^{1,2,3}$
}

\begin{abstract}
Sepsis-induced multiple organ dysfunction and inflammatory response are life-threatening symptoms without effective treatment. Fisetin, a dietary flavonoid extracted from berries and family Fabaceae, has displayed neuroprotective and anti-oxidant activities. In this study we investigated whether fisetin exerted a protective effect against sepsis-induced multiple organ dysfunction in mouse cecum ligation and puncture (CLP) model. The mice were injected with fisetin (10 mg/kg, ip) $0.5 \mathrm{~h}$ prior to CLP, and sacrificed $18 \mathrm{~h}$ after CLP. We found that fisetin administration significantly alleviated CLP-induced lung, liver and kidney injury, as well as the expression levels of interleukin (IL)-6, tumor necrosis factor (TNF)- $\alpha$ and IL-1 $\beta$ in bronchoalveolar lavage fluid (BALF). In lipopolysaccharide (LPS)-treated mouse bone marrow-derived macrophages (BMDMs), application of fisetin (3-10 $\mu \mathrm{M})$ dosedependently inhibited the expression levels of IL-6, TNF- $a, I L-1 \beta$, and inducible nitric oxide synthase (iNOS). Furthermore, fisetin dose-dependently inhibited the phosphorylation of p38 MAPK, MK2, and transforming growth factor- $\beta$-activated kinase (TAK) 1 via attenuating the interaction between TAK1 and TAK-binding proteins (TAB) 1 . These results demonstrate that fisetin is a promising agent for protecting against sepsis-induced inflammatory response and organ injury via inhibiting macrophage activation.
\end{abstract}

Keywords: Fisetin; sepsis; multiple organ dysfunction; inflammation; mouse cecum ligation and puncture (CLP) model; macrophages

Acta Pharmacologica Sinica (2020) 41:1348-1356; https://doi.org/10.1038/s41401-020-0462-y

\section{INTRODUCTION}

Sepsis involves systemic inflammation with multiple organ dysfunction that causes high morbidity and mortality in clinical patients [1]. Generally, a considerable proportion of patients in intensive care units (ICUs) are affected by sepsis [2-4]. Although medical applications for treating septic infection have been improved, the mortality of patients is still higher than $25 \%$ and reaches $40 \%-50 \%$ in cases of septic shock $[5,6]$. Therefore, developing promising drug candidates with specific and effective therapies is an important topic for patients with sepsis and septic symptoms.

The acute inflammatory response is one of the causes of the high mortality in patients with sepsis [7]. An overactivated inflammatory response with dysregulated expression of inflammatory factors during the early stage of sepsis has been regarded as the leading cause of dysfunction of multiple organs, including lung, liver, and kidney injury [7, 8]. Proinflammatory cytokines, such as interleukin (IL)- 6 , tumor necrosis factor (TNF)- $\alpha$, and IL-1 $\beta$, and nitric oxide (NO) directly induce tissue injury and initiate neutrophil infiltration. Therefore, inhibiting the inflammatory response during sepsis can prevent or reduce organ injury $[9,10]$.

The innate immune system has a significant effect on inflammation and sepsis-induced organ injury. As an important component of innate immunity, macrophages are critical for mediating the inflammatory response and are also involved in the regulation of sepsis [11]. Inflammatory factors produced by macrophages, including IL-1 $\beta, I L-6$, and TNF- $\alpha$, directly induce organ injury and participate in regulating homeostasis through phagocytosis and erythropoiesis [12-14].

The identification of pathogen-associated molecular patterns (PMAPs) by pattern recognition receptors can lead to the transformation of extracellular stimuli into intracellular signals [15-17]. Toll-like receptors (TLRs) are major receptors on macrophages that combine with internal pathological mediators to respond to excess pathogenic stimulation [17]. For example, lipopolysaccharide (LPS), an endotoxin from gram-negative bacteria, binds to TLR4, resulting in the excessive production of proinflammatory factors, such as NO and TNF-a [18]. LPS is a primary stimulus that activates macrophages and has been commonly used in the study of sepsis pathogenesis. After challenge with LPS, transforming growth factor- $\beta$-activated kinase (TAK) is activated upon binding with TAK-binding proteins (TAB), resulting in the promotion of the nuclear translocation and activation of nuclear factor kappa B (NF-KB) and mitogenactivated protein kinase (MAPK) signaling pathways [19-21]. Therefore, interference of these signaling pathways may inhibit macrophage activation and reduce the inflammatory response during sepsis.

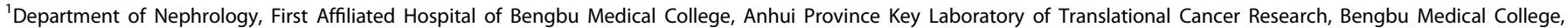

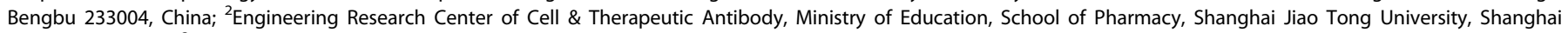
200240, China and ${ }^{3}$ Jiangsu Center for the Collaboration and Innovation of Cancer Biotherapy, Cancer Institute, Xuzhou Medical University, Xuzhou 221004, China

Correspondence: Feng Qian (fengqian@sjtu.edu.cn)

These authors contributed equally: Hai-feng Zhang, Hai-bo Zhang

Received: 24 March 2020 Accepted: 11 June 2020

Published online: 13 July 2020 
Fisetin, a dietary flavonoid extracted from fruits, such as berries, and members of the Fabaceae family [22], is well known for its neuroprotective, antioxidant and anticancer effects manifesting through a complex signaling networks [23-25]. In the prevention of an inflammatory response, fisetin attenuates IL-1 $\beta$-mediated epithelial cell injury and decreases the expression of cytokines IL- 6 and TNF- $a$ and chemokines [26]. In addition, fisetin protects against sepsis-induced multiple organ failure by inhibiting macrophage activation.

In the present study, the protective role of fisetin in aseptic model of mice subjected to cecal ligation and puncture (CLP) was determined. Fisetin significantly reduced the expression of inflammatory cytokines and ameliorated sepsis-induced multiple organ failure by attenuating lung, liver, and kidney injury in vivo. In addition, fisetin inhibited the production of IL-1 $\beta$, IL-6, and TNF$a$ and suppressed TAK1/p38MAPK/MK2 signaling pathways. Our study revealed the anti-inflammatory effect of fisetin, indicating that fisetin is a promising agent for preventing sepsis-induced organ injury.

\section{MATERIALS AND METHODS}

Materials and reagents

Fisetin $\left(\mathrm{C}_{15} \mathrm{H}_{10} \mathrm{O}_{6}\right.$, MW 286.24) was purchased from Target Mol Co., Ltd. (Shanghai, China), and its purity was greater than $98 \%$, as confirmed by HPLC. Fisetin was dissolved in DMSO and stored at $-20^{\circ} \mathrm{C}$. LPS (Cat. no. L4391) and DMSO (Cat. no. D2650) were obtained from Sigma (St Louis, MO, USA). Dulbecco's modified Eagle's medium (DMEM, Cat. no. 88284), antibiotics (100 units $/ \mathrm{mL}$ penicillin and $0.1 \mathrm{mg} / \mathrm{mL}$ streptomycin, Cat. no. 10131027), $0.25 \%$ trypsin (Cat. no. 25200056) and fetal bovine serum (FBS, Cat. no. 16140071) were purchased from Life Technologies (Grand Island, NY, USA). The ReverTra Ace qPCR TR kit (Cat. no. FSQ-101) and THUNDERBIRD SYBR qPCR Mix (Cat. QPS-201B) were purchased from Toyobo (Osaka, Japan). Recombinant murine macrophage colony-stimulating factor (M-CSF) was obtained from PeproTech (Rocky Hill, NJ, Cat. no. 315-02). A BCA protein assay kit (Cat. no. P00125) and RIPA lysis buffer (Cat. P0013K) were purchased from Beyotime Biotechnology (Shanghai, China). Western blotting antibodies, including anti-p-p38 MAPK (Cat. no. 4511), anti-p38 MAPK (Cat. 8690), anti-p-MK2 (Cat. no. 3041), anti-MK2 (Cat. no. 3042), anti- $\beta$-actin (Cat. no. 4970), anti-p-TAK1 (Cat. no. 9339), antiTAK1 (Cat. no. 5206), anti-Flag-Tag (Cat. no. 14793) and anti-MycTag (Cat. no. 2276) antibodies were purchased from Cell Signaling Technology (Danvers, MA, USA). Antibodies to detect inducible nitric oxide synthase (iNOS) were obtained from Santa Cruz Biotechnology (California, USA, Cat. no. sc-7271). Alanine aminotransferase (ALT, Cat. no. C009-2), aspartate aminotransferase (AST, Cat. no. C010-2), blood urea nitrogen (BUN, Cat. no. C013-2) and serum creatinine detection kits (Cat. no. C011-2) were purchased from Nanjing Jiancheng Bioengineering Institute (Nanjing, China). Fluorescein isothiocyanate (FITC)-conjugated anti-mouse Gr-1 (Ly-6G) antibody (Cat. no. RM3001) and the enhanced chemiluminescence (ECL) kit (Cat. no. 35050) were obtained from eBioscience by Thermo Fisher Scientific (Grand Island, NY, USA). Enzyme-linked immunosorbent assay (ELISA) kits for mouse IL-1 $\beta$ (Cat. no. MLB00C), IL-6 (Cat. no. D6050) and TNF-a (Cat. no. MTA00B) were purchased from R\&D Systems (Minneapolis, MN, USA). Other chemical reagents were purchased from Sigma unless stated otherwise.

Cecal ligation and puncture-induced sepsis model

Cecal ligation and puncture (CLP) was used to induce murine sepsis. Male C57BL/6 mice (6-8 weeks old) with an average weight of $20-25 \mathrm{~g}$ were purchased from SLAC Animal Company (Shanghai, China) and maintained in 12-h light and 12-h dark cycles with free access to food and water. The mice were randomly separated into 3 groups ( $n=6$ per group): (1) sham group; (2) CLP + vehicle (DMSO, $1 \mu \mathrm{L} / \mathrm{g}$ ); and (3) CLP + fisetin (10 $\mathrm{mg} / \mathrm{kg}$, dissolved in $1 \mu \mathrm{L} / \mathrm{g}$ DMSO). Briefly, the mice were anesthetized by pentobarbital sodium. Then, a midline laparotomy was performed, and the cecum was exteriorized and ligated distal to the ileocecal valve (without causing intestinal obstruction). The cecum was perforated using a 20-gauge needle and squeezed gently to extrude a small amount of fecal contents from the punctured cecum. The cecum was then returned to the peritoneal cavity, and the incision was closed using two layers of sutures. In the sham group, animals were anesthetized, and the cecum was exteriorized without ligation or puncture. In the CLP + vehicle group, the animals were intraperitoneally injected with vehicle $0.5 \mathrm{~h}$ before the CLP procedure was performed. In the CLP + fisetin group, the animals were intraperitoneally injected with fisetin $0.5 \mathrm{~h}$ before the CLP procedure was performed. All animals were sacrificed $18 \mathrm{~h}$ after the CLP procedure. Lung tissues, kidney tissues, liver tissue, bronchoalveolar lavage fluid (BALF), and blood were collected for further analysis. All experimental procedures mentioned in this study were approved by the Animal Care Use Committee of Bengbu Medical College. The animal experiments were performed in accordance with the National Institutes of Health Guide for the Care and Use of Laboratory Animals.

\section{Myeloperoxidase (MPO) activity assay}

MPO activity was measured to evaluate tissue neutrophil accumulation. The lung tissues and liver tissues were homogenized and suspended in $0.5 \%$ hexadecyltrimethylammonium bromide (HTAB) buffer. The mixture was centrifuged and treated with a freeze-thaw procedure. The protein concentration of the supernatant was determined using a BCA protein quantitation kit. The MPO activity was measured by monitoring the change in absorbance at $655 \mathrm{~nm} 5 \mathrm{~min}$ after 3,3',5,5'-tetramethylbenzidine (TMB) and $\mathrm{H}_{2} \mathrm{O}_{2}$ were added to the tissue samples.

\section{Pulmonary histological evaluation}

The mice were sacrificed after the CLP procedure and treatment with fisetin. Part of the pulmonary tissue samples were fixed with $4 \%$ paraformaldehyde for $48 \mathrm{~h}$, dehydrated in graded alcohol, embedded in paraffin wax, and stained with hematoxylin-eosin for histomorphometric assessment. The histological sections were photographed using light microscopy.

Neutrophil aggregation assay

The red blood cells in the BALF were lysed using lysis buffer and then centrifuged. The remaining cells in the BALF were washed slightly, resuspended in PBS and counted. The neutrophils were labeled with anti-mouse Ly-6G (Gr-1)-FITC antibody for $30 \mathrm{~min}$ at $4{ }^{\circ} \mathrm{C}$. The samples were measured by a FACSCanto flow cytometer (Becton Dickinson). The data were analyzed using FlowJo 7.6 (Tree Star, Ashland OR, USA) software.

\section{Assessment of liver and kidney functions}

Blood samples were collected through a cardiac puncture and centrifuged at $1000 \times g$ for $5 \mathrm{~min}$ to separate serum. Serum alanine aminotransferase (ALT), aspartate aminotransferase (AST), blood urea nitrogen (BUN), and creatinine were measured with detection kits according to the manufacturers' instructions (Nanjing Jiancheng Bioengineering Institute).

\section{Reverse-transcription PCR}

Total RNA was extracted from BMDMs or homogenized lung tissues using TRIzol reagent (Invitrogen) and reverse-transcribed to cDNA using a ReverTra Ace qPCR RT kit. The StepOnePlus System (Thermo Fisher Scientific, Waltham, MA, USA) was used for real-time $P C R$ with THUNDERBIRD SYBR $\mathrm{qPCR}$ mix for the quantification of the CDNA. The following gene-specific primers were used: NGAL, forward 5'-CTCAGAACTTGATCCCT-GCC-3' and reverse $5^{\prime}$-TCCTTGAGGCCCAGAGACTT-3'; KIM-1, forward 5'-TGCT 
G-CTACTGCTCCTTGTG-3' and reverse $5^{\prime}$-GGGCCACTGG TACTCATT CT-3'; IL-6, forward 5'-CCACCAAGAACGATAGTCAA-3' and reverse 5'-TTCCACGATTCCC-AGA-3'; TNF-a, forward 5'-TTCTCATTCCTG CTTGTGG-3' and reverse $5^{\prime}$-ACTTGGT-GGTTGCTACG-3'; IL-1 $\beta$, forward $5^{\prime}$-CCAGCTTCAAATC TCACAGCAG-3' and reverse $5^{\prime}$-CTTCT TTGGGTATTGCTTGGGATC-3'; GAPDH, forward 5'-TGCGACTT-CAA CAGCAACTC-3' and reverse $5^{\prime}$-CTTGCTCAGTGTCCT TGCTG-3'.

Enzyme-linked immunosorbent assays (ELISAs)

ELISAs were used to measure the concentrations of the different cytokines and chemokines. The concentrations of TNF- $\alpha, I L-1 \beta$, and IL- 6 in the supernatant from the BMDMs and BALF were measured using ELISA kits according to the manufacturers' instructions.

\section{Cell isolation and culture}

Murine bone marrow was collected from C57BL/6 mice and cultured in DMEM with $10 \%$ FBS and $1 \%$ streptomycin-penicillin. The cells were treated with $10 \mathrm{ng} / \mathrm{mL}$ M-CSF for 7 days to obtain bone marrow-derived macrophages (BMDMs). HEK293T cells were purchased from American Type Culture Collection (ATCC, Manassas, VA) and cultured in DMEM supplemented with $10 \%$ FBS and $1 \%$ streptomycin-penicillin. All the cells were maintained at $37^{\circ} \mathrm{C}$ in $5 \% \mathrm{CO}_{2}$.

\section{Quantitative determination of the nitrite levels}

The Griess reagent was used to determine the nitrite levels. BMDMs were treated with fisetin at $0,3,10$, or $30 \mu \mathrm{M}$ for $30 \mathrm{~min}$ followed by challenge with $100 \mathrm{ng} / \mathrm{mL}$ LPS for $12 \mathrm{~h}$. The cell culture supernatant was collected, and Griess reagent was added. The nitric oxide (NO) levels were measured using a microplate reader (Flex Station 3, Molecular Devices, USA) at a wavelength of $540 \mathrm{~nm}$.

\section{Western blotting analysis}

Western blot assays were performed to determine protein expression. Whole-cell lysates were prepared using RIPA lysis buffer. After boiling for $10 \mathrm{~min}$, the proteins were separated on $10 \%$ SDS-PAGE gels and transferred to $0.45 \mu \mathrm{M}$ of $\mathrm{NC}$ membranes. The membranes were blocked with $5 \%$ skim milk at room temperature and probed with primary antibodies overnight at $4{ }^{\circ} \mathrm{C}$, followed by incubation with HRP-conjugated secondary antibodies. The protein signals were detected using an ECL kit. Quantitative analysis was accomplished with ImageJ software (National Institute of Mental Health, Bethesda, MD, USA).

\section{Luciferase reporter assay}

The relative NF-KB activity was analyzed using a luciferase reporter assay. All plasmids were purchased from Shanghai HuaGene biotech Co., Ltd. (Shanghai, China). HEK293T cells were plated on 24-well plates and transfected with $0.5 \mu \mathrm{g}$ of NF-KB luciferase reporter plasmid, $2.5 \mu \mathrm{g}$ of vector DNA or plasmid DNA (TAK1/ TAB1) using $6 \mu \mathrm{g}$ of polyethyleneimine (PEI) according to the manufacturer's instructions. Eighteen hours after transfection, fisetin $(0,3,10$, or $30 \mu \mathrm{M})$ was added to the cells, and the relative NF-кB activity was determined $6 \mathrm{~h}$ later.

\section{Co-Immunoprecipitation (Co-IP)}

HEK293T cells were plated on 6-well plates and transfected with different plasmids (Flag-TAK1 and Myc-TAB1), which were purchased from Shanghai HuaGene biotech Co., Ltd. (Shanghai, China) using polyethyleneimine (PEI). Forty-two hours after transfection, $10 \mu \mathrm{M}$ fisetin was added. After incubation for another $8 \mathrm{~h}$, the HEK293T cells were lysed with cold RIPA lysis buffer. The cell lysates were incubated with specific antibodies overnight, and protein $\mathrm{G}$ beads were subsequently added. After washing, the coIP samples were analyzed by immunoblotting.
Statistical analysis

Data are presented as the means \pm SEM. One-way ANOVA, followed by Student's $t$-test, was used to compare the data among multiple groups. Statistical analysis was performed and histograms were generated using Prism software (ver. 6.2; GraphPad, San Diego, CA). $P<0.05$ was considered a significant difference.

\section{RESULTS}

Fisetin ameliorates CLP-induced acute lung injury

To determine the effects of fisetin on sepsis-induced acute lung injury, a mouse CLP model was established, and the fisetin treatment procedures are shown in Fig. 1a. CLP-induced lung injury in the mice was determined by histological analysis. CLPinduced pathologic reorganization in lung lobes was observed, which also presented with severe alveolar-capillary structure damage and inflammatory cell infiltration based on H\&E staining. Compared with effects in the CLP group, the collapse of alveolar spaces and the infiltration of inflammatory cells were largely ameliorated in the mice treated with fisetin (Fig. 1b). To determine whether fisetin attenuated pulmonary neutrophil infiltration, we measured the proportion of neutrophils in the BALF from the CLP mice (Fig. 1c). The infiltrative neutrophils were largely reduced in the mice treated with fisetin compared with those in the CLP group (Fig. 1d). An MPO analysis was also performed to examine neutrophil infiltration into the lung tissue. As expected, MPO activity was markedly attenuated after treatment with fisetin (Fig. 1e). Consistent with the data presented above, the wet-to-dry ratio of the lung tissue was elevated after CLP and was found to be reduced in the fisetin group (Fig. 1f). The concentration of total proteins in the BALF was increased in the CLP group but decreased in the fisetin group (Fig. 1g). These results indicate that fisetin mitigated the CLP-induced inflammatory response and acute lung injury.

Fisetin alleviates CLP-induced liver and kidney injury The levels of ALT and AST in serum are important indexes for evaluating the degree of liver injury induced by CLP. The values of ALT and AST increased approximately fivefold in the mice that subjected to the CLP operation. However, the levels of ALT and AST were remarkably reduced in the fisetin group (Fig. 2a, b). In addition, liver tissues were homogenized, and MPO analysis was performed. MPO activity was enhanced after the CLP procedure and was lower in the fisetin group (Fig. 2c). As typical markers of kidney injury, the expression of BUN and creatinine in serum, were measured. The results indicated that the levels of BUN and creatinine in serum were remarkably increased after the CLP operation, but the finding was reversed in the fisetin group (Fig. 2d, e). Furthermore, changes in the mRNA levels of neutrophil gelatinase-associated lipocalin (NGAL) and kidney injury molecule1 (KIM-1) showed a relatively consistent trend of fisetin inhibiting their expression (Fig. 2f, g). Therefore, the results suggest that fisetin alleviated CLP-induced liver and kidney injury.

Fisetin attenuates inflammatory cytokine production in vivo To determine the effects of fisetin on inflammation, we measured the protein levels of proinflammatory cytokines IL-6, TNF- $a$, and IL$1 \beta$ in the BALF by using ELISAs (Fig. $3 a-c$ ). Mice subjected to the CLP operation expressed high levels of these proinflammatory cytokines. However, the levels were significantly reduced after fisetin treatment. These results suggest fisetin attenuated inflammatory cytokine production in the CLP model.

Fisetin reduces the expression of proinflammatory cytokines in the BMDMs

To determine the effects of fisetin on the LPS-induced inflammatory response in vitro, BMDMs were pretreated with fisetin at 0,3 , 
a

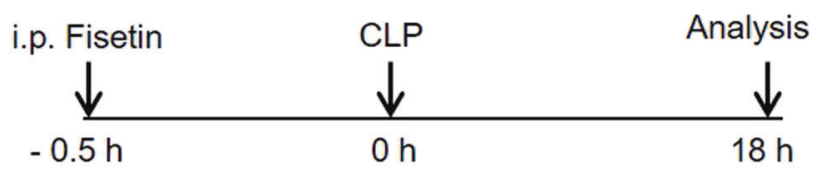

b

Sham

CLP + Vehicle

CLP + Fisetin

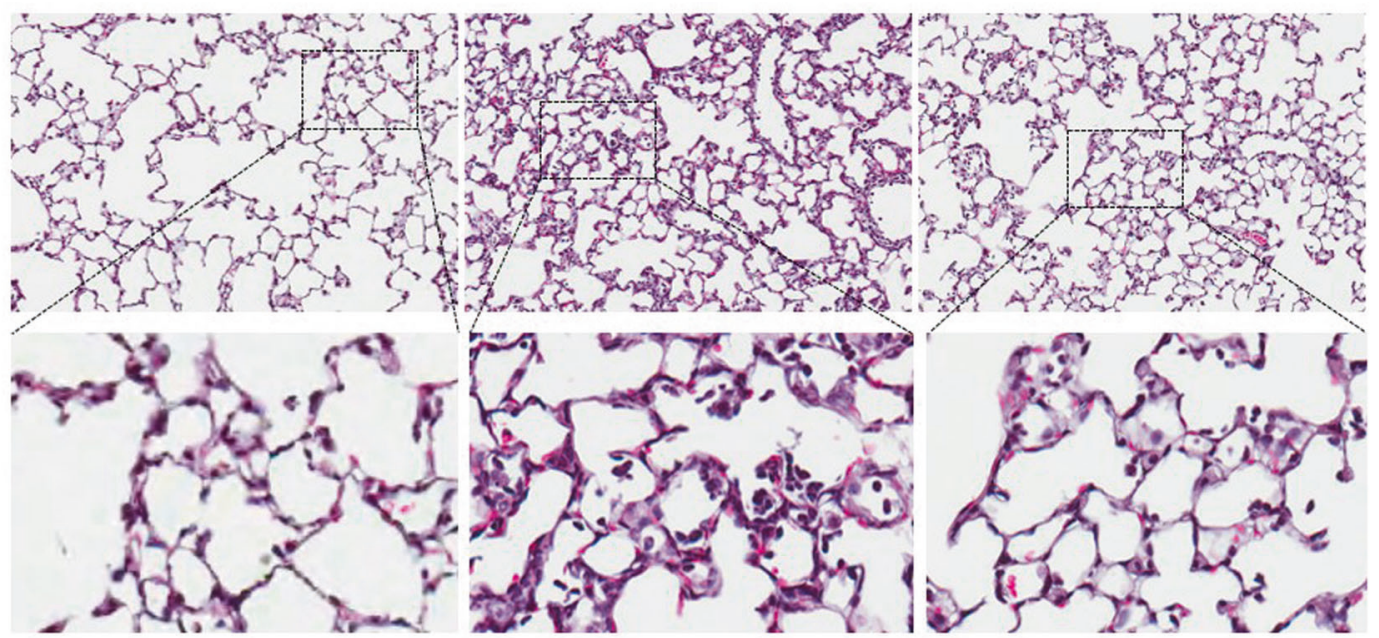

C

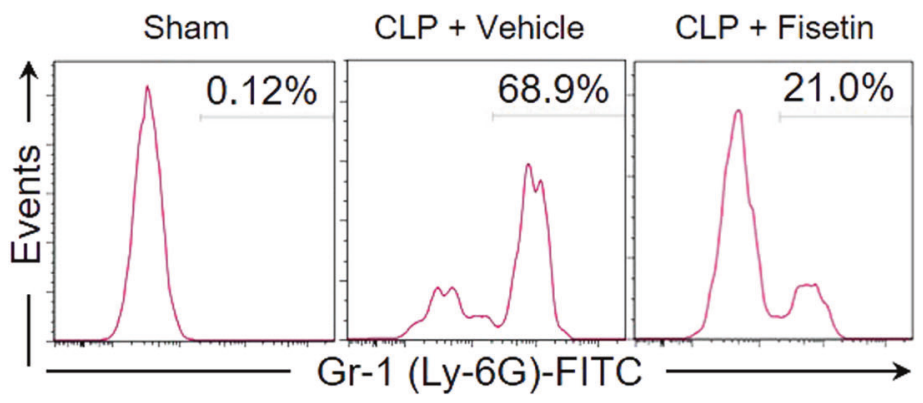

d

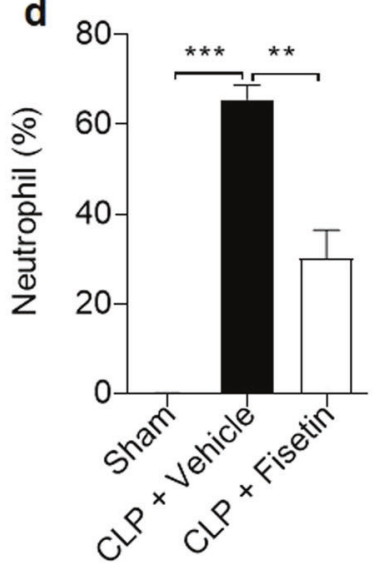

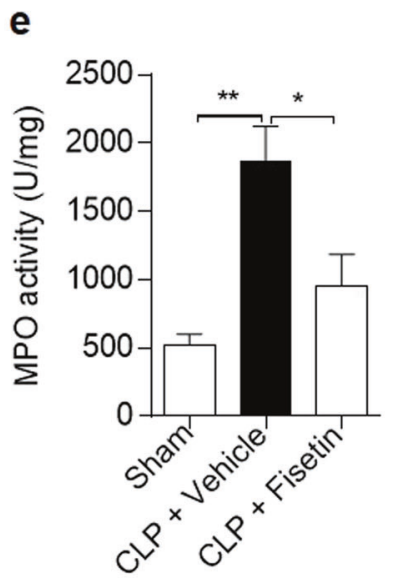

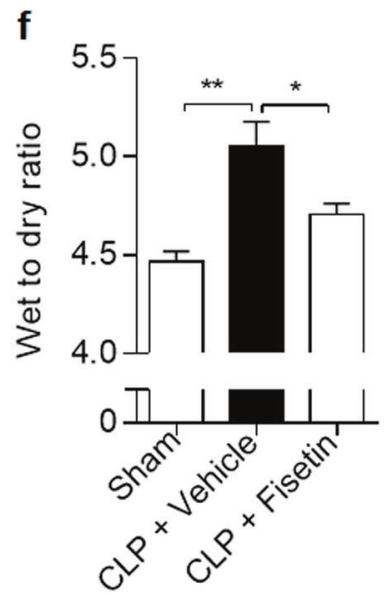

g

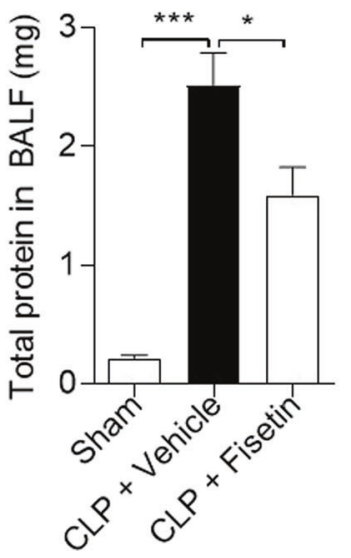

Fig. 1 Fisetin ameliorates CLP-induced acute lung injury. Animals were intraperitoneally injected with $10 \mathrm{mg} / \mathrm{kg}$ fisetin $0.5 \mathrm{~h}$ before CLP surgery. The mice were sacrificed, and BALF and lung tissues were collected $18 \mathrm{~h}$ after the CLP procedure. a A brief summary of the procedures used in the animal experiments is shown. b H\&E staining of lung tissues was performed (20x, upper and 40x, lower). c Flow cytometry analysis of the neutrophil percentage in BALF. The FITC-positive cells were neutrophils. $\mathbf{d}$ Statistical analysis showing the neutrophil percentage in a histogram. e MPO activity in lung tissue homogenates was measured. $\mathbf{f}$ Wet-to-dry ratios of lung tissues were measured. g Total protein concentrations in BALF were tested. Results are expressed as the means $\pm \mathrm{SEM}, n=6$ mice per group $\left(^{*} P<0.05,{ }^{* *} P<0.01\right.$, and $* * * P<0.001)$. 

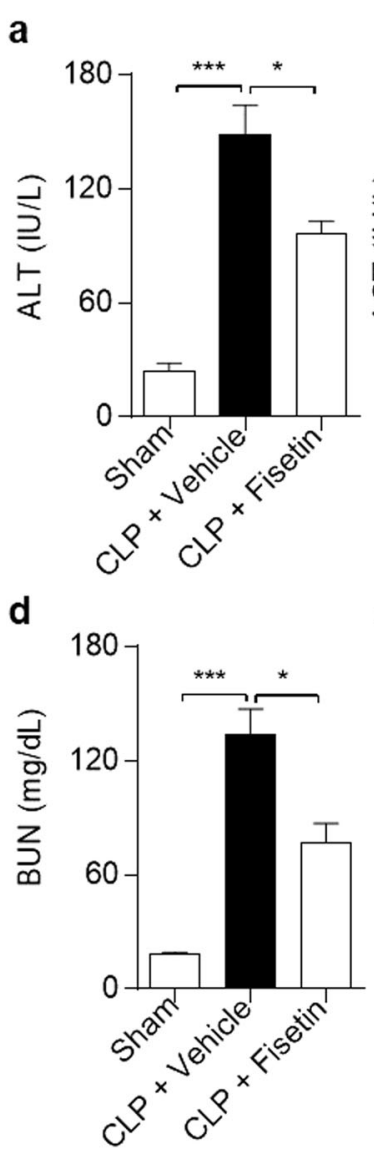
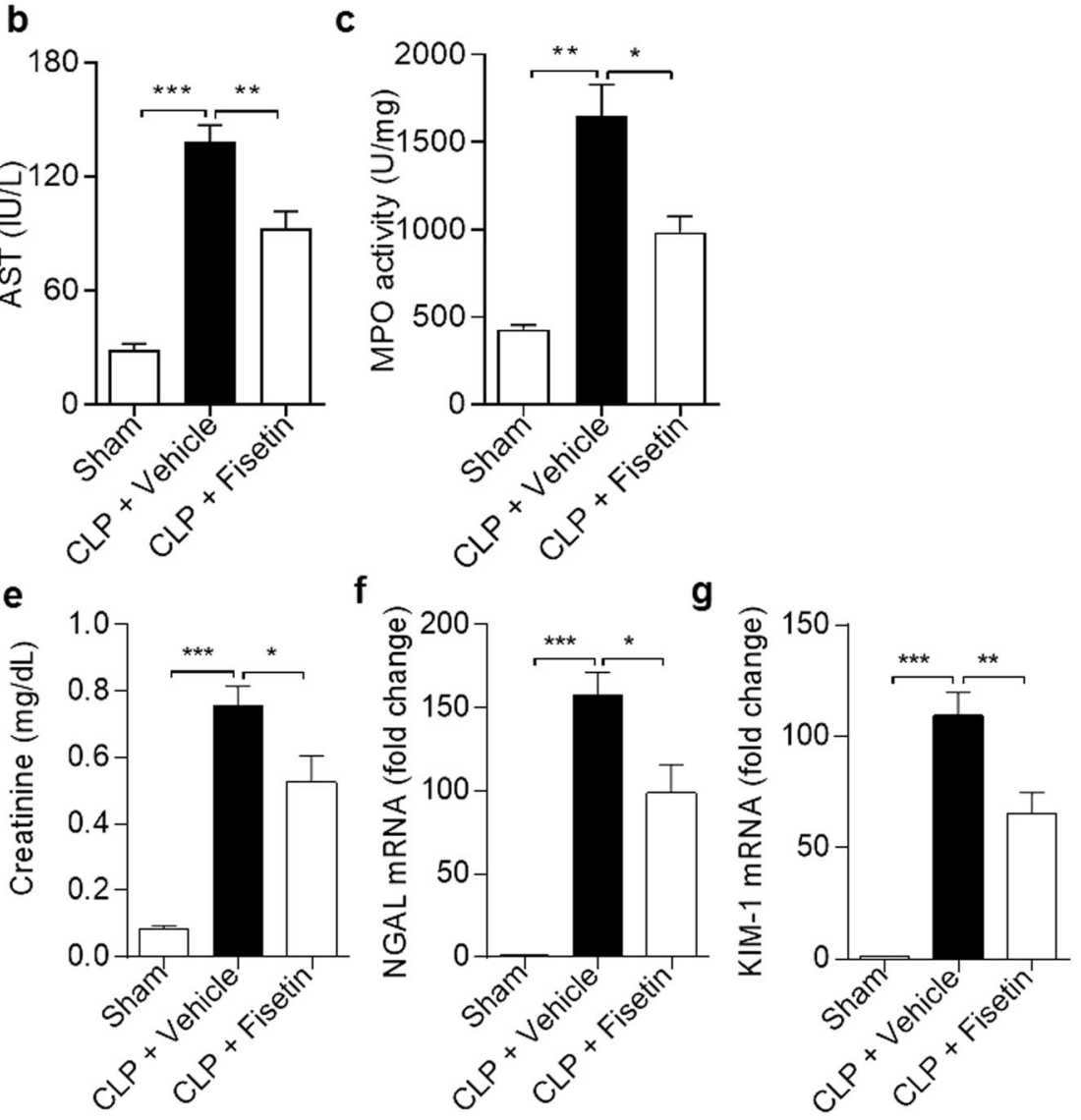

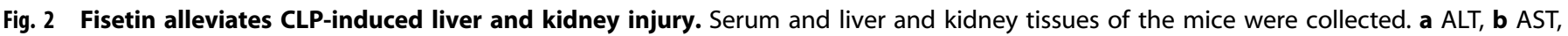

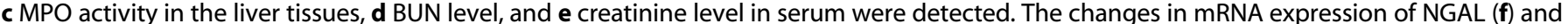

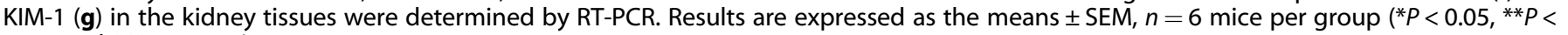
0.01 , and $\left.{ }^{* * *} P<0.001\right)$.
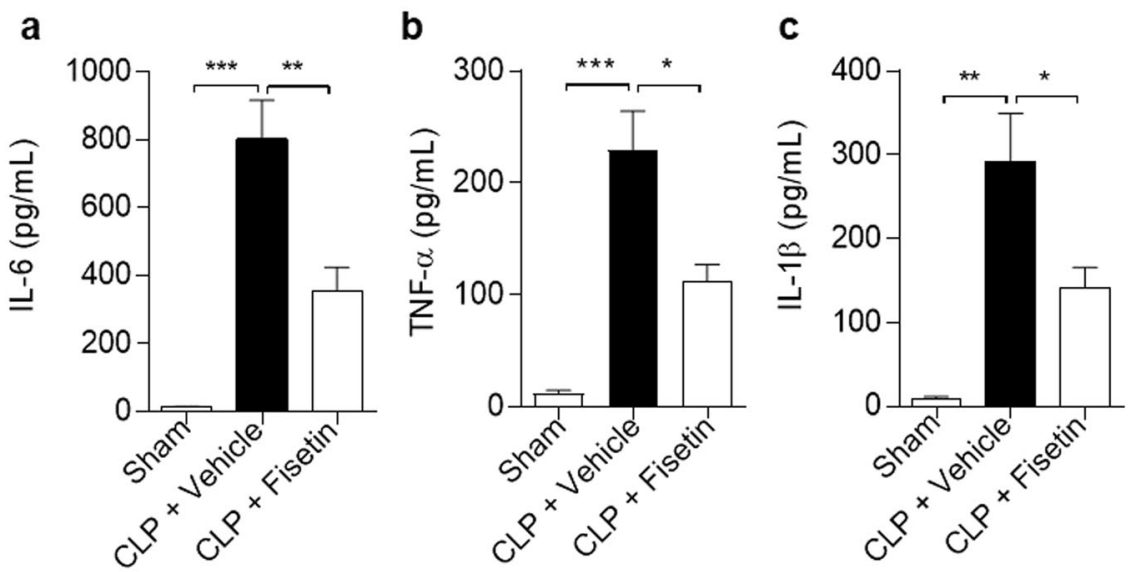

Fig. 3 Fisetin attenuates inflammatory cytokine production. The changes in cytokine expression of (a) IL-6, (b) TNF- $\alpha$, and (c) IL-1 $\beta$ in BALF were detected by ELISA. The results are expressed as the mean \pm SEM, $n=6$ mice per group $\left({ }^{*} P<0.05,{ }^{* *} P<0.01\right.$, and $\left.{ }^{* * *} P<0.001\right)$.

10 , or $30 \mu \mathrm{M}$ for 30 min before stimulation with $100 \mathrm{ng} / \mathrm{mL}$ LPS for $12 \mathrm{~h}$. The proinflammatory cytokine production was measured by ELISAs. The levels of IL- 6 , TNF- $a$, and IL-1 $\beta$ in the supernatants of the LPS group were dramatically increased compared with that of the control group, while the levels of cytokine production were reduced in the fisetin group in a dose-dependent manner (Fig. 4a-c). These findings suggest that fisetin suppressed inflammatory cytokine production in vitro.
Fisetin inhibits the expression of iNOS and NO production in the BMDMs

To revaluate the effects of fisetin on LPS-induced inflammation in vitro, the expression of iNOS was measured. As shown in Fig. 5, iNOS was inhibited in the fisetin group in a dose-dependent manner, compared with that of the LPS group in the BMDMs (Fig. 5a, c). Furthermore, the generation of NO in the supernatant was measured by the Griess reagent. The level of NO was 


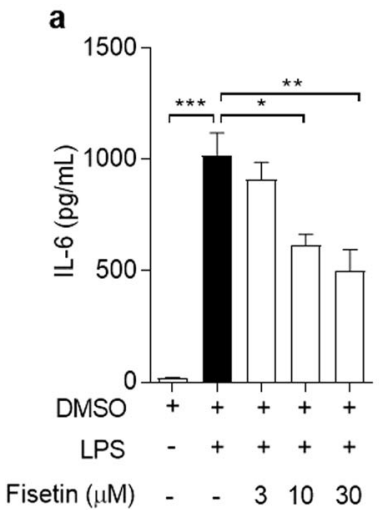

b

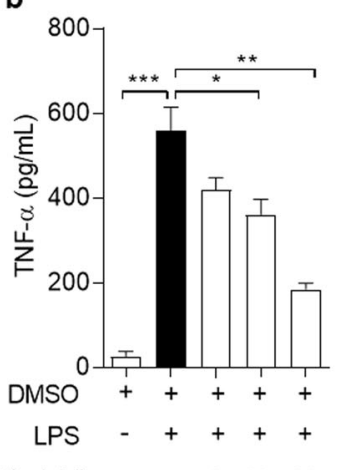

c

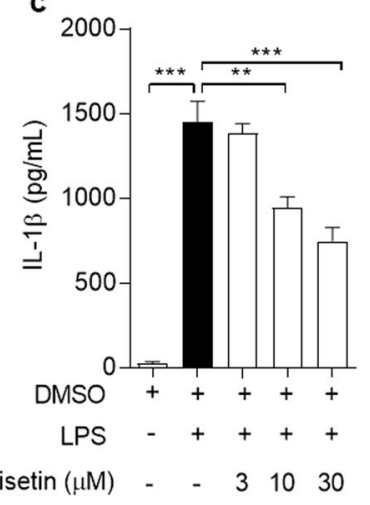

Fig. 4 Fisetin decreased the expression of proinflammatory cytokines in the BMDMs. BMDMs were treated with fisetin at $0,3,10$, or $30 \mu \mathrm{M}$ for $30 \mathrm{~min}$ before stimulation with $100 \mathrm{ng} / \mathrm{ml}$ LPS for $12 \mathrm{~h}$. The expressions of cytokines: $\mathbf{a} \mathrm{IL}-6, \mathbf{b}$ TNF- $\alpha$, and $\mathbf{c}$ IL-1 $\beta$ in the supernatants of the BMDMs, as detected by ELISAs. Results are expressed as the means \pm SEM of three independent experiments $\left({ }^{*} P<0.05\right.$, ${ }^{* *} P<0.01$, and ${ }^{* * *} P<$ 0.001).

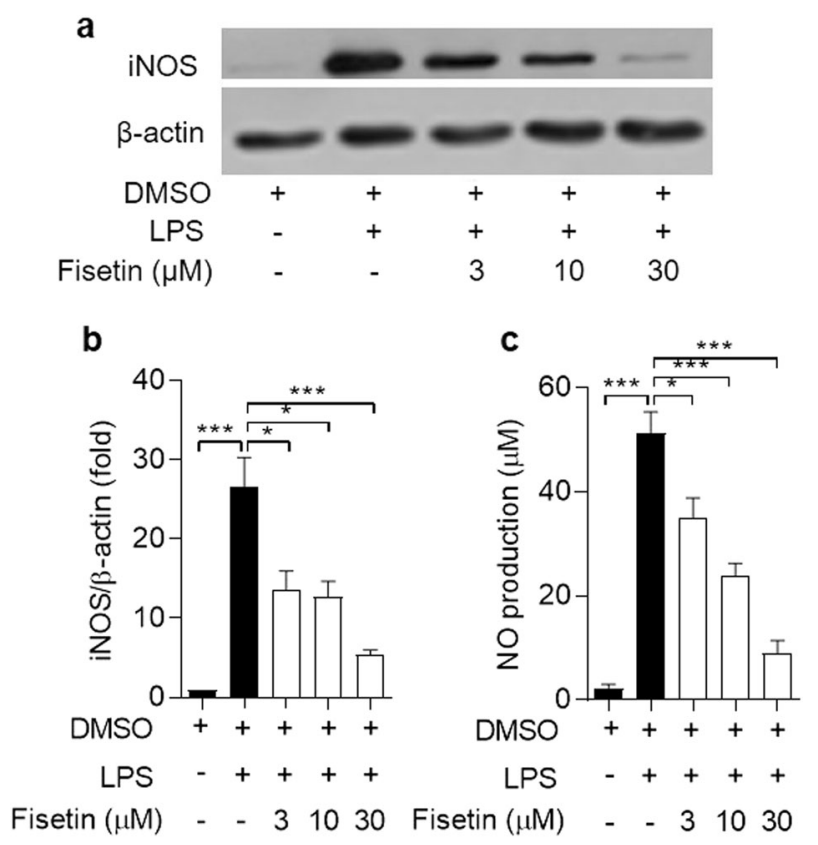

Fig. 5 Fisetin inhibits the production of iNOS and NO in the BMDMs. a The protein expression of iNOS and $\beta$-actin in the BMDMs was determined by Western blotting. b Statistical analysis of iNOS expression normalized to $\beta$-actin is shown in the histogram. c NO production in the BMDMs was measured. The results are expressed as the means \pm SEM of three independent experiments $\left({ }^{*} P<0.05\right.$ and ${ }^{* * *} P<0.001$ )

markedly induced by LPS and inhibited by fisetin in a dosedependent manner (Fig. 5c). These results indicate that fisetin inhibited iNOS and NO expression.

Fisetin downregulates p38/MK2 signaling pathway in the BMDMs The MAPK signaling pathway plays an important role in macrophage activation and proinflammatory cytokine production. Therefore, we determined whether fisetin ameliorated LPSinduced macrophage activation by regulating the MAPK signaling pathway. As shown in Fig. 6a, the phosphorylation of p38 was increased after stimulation with LPS. The LPS-induced phosphorylation of p38 MAPK was reduced by fisetin in a dosedependent manner (Fig. 6b). To explore the effect of fisetin on the p38 MAPK signaling pathway, we measured the activity of
MK2, the downstream kinase of p38 MAPK. The phosphorylation of MK2 was significantly downregulated by fisetin (Fig. 6c). Altogether, fisetin exerted anti-inflammatory effects by downregulating the activation of the p38MAPK/MK2 signaling pathway.

Fisetin suppresses NF-KB and p38 activity by targeting TAK1/TAB1 To determine how fisetin suppresses the p38 MAPK/ MK2 signaling pathway, we measured the activation of TAK1, a signal transducer upstream of p38 MAPK and NF-KB. We first researched whether fisetin inhibited the phosphorylation of TAK1 in the BMDMs. LPS-induced phosphorylation of TAK1 was reduced by fisetin in a dose-dependent manner (Fig. 7a, b). The phosphorylation of TAK1 was regulated by the formation of the TAK1-TAB1 complex. We then cotransfected HEK293T cells with Flag-TAK1 and Myc-TAB1. As shown in Fig. 7c, d, fisetin significantly inhibited the interaction between TAK 1 and TAB1. Furthermore, we cotransfected the NF-KB luciferase reporter with TAK1/TAB1 plasmids into HEK293T cells. After the cells were treated with $0,3,10$, or $30 \mu \mathrm{M}$ fisetin for $6 \mathrm{~h}$, the luciferase activity levels were measured. As shown in Fig. 7d, fisetin effectively suppressed the activity of the NF-KB luciferase reporter driven by TAK1/TAB1. These results suggest that fisetin attenuates TAK1-mediated MAPK activation by interfering with the interaction between TAK1 and TAB1.

\section{DISCUSSION}

Severe sepsis, including septic shock and sepsis-induced multiple organ failure, is a major cause of death in ICUs. Acute lung injury (ALI) and liver and kidney injury are the major organs involved in sepsis-induced multiple organ failure. The pathological environment of sepsis-induced multiple organ failure is often compromised by complex factors, such as inflammation, damaged vascular endothelial injury, robust activation of complements, and increased oxidative stress [27, 28]. Macrophages are important regulatory cells in the sepsis-related inflammatory response because they produce proinflammatory factors and mediators [29]. Macrophages are able to release large numbers of inflammatory factors, such as IL-1 $\beta$, TNF- $a$, and IL-6, after CLP or LPS treatment $[30,31]$. In addition, overactivated inflammation causes acute systemic organ injuries during the early stage of organ failure. For example, TNF-a recruits inflammatory cells, leading to the release and accumulation of proinflammatory cytokines and inducing necroptosis because of a local auto amplification loop and solid organ failure $[32,33]$. Applying anti-TNF-a antibodies remarkably suppresses 
a

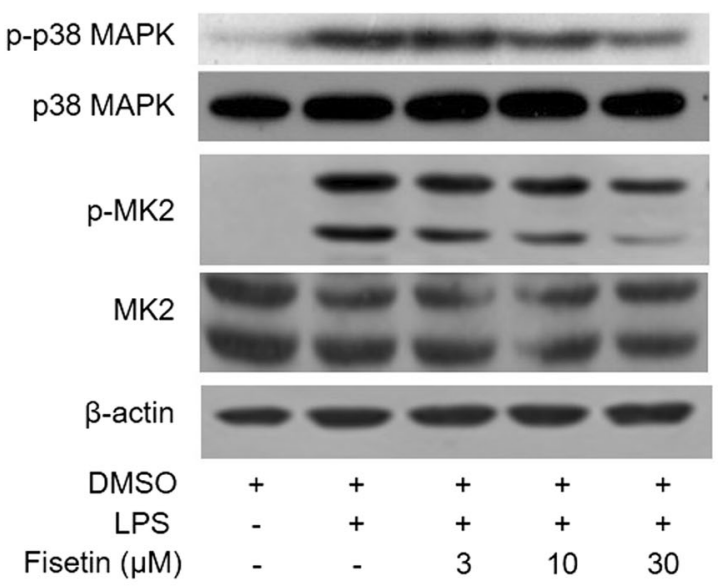

b

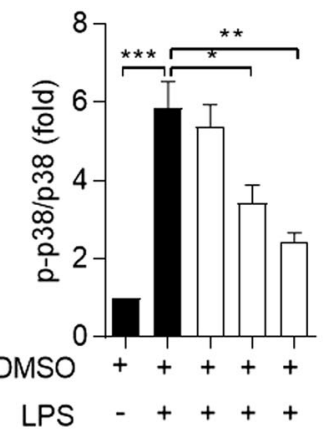

Fisetin $(\mu \mathrm{M})-31030$

C

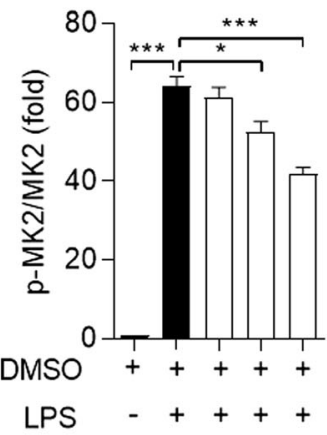

Fisetin $(\mu \mathrm{M}) \quad-\quad-31030$

Fig. 6 Fisetin downregulates the p38 MAPK/MK2 signaling pathway in the BMDMs. a The protein expression levels of p-p38 MAPK, p38MAPK, p-MK2, MK2, and $\beta$-actin were evaluated by Western blotting. Statistical analysis of (b) p-p38 and (c) p-MK2, as shown in the histograms. The results are expressed as the means \pm SEM of three independent experiments $\left({ }^{*} P<0.05,{ }^{* *} P<0.01\right.$, and $\left.{ }^{* * *} P<0.001\right)$.

inflammation and increases the survival rate of patients with severe sepsis [34]. Macrophages also have the ability to produce NO by inducing the expression of iNOS in response to LPS challenge [35]. iNOS-knockout mice showed decreased mortality in late sepsis, and selective iNOS inhibition alleviated sepsisinduced acute kidney injury [36]. Here, we found that the expression of IL-1 $\beta$, TNF- $a$, and IL- 6 was significantly reduced by fisetin pretreatment of the CLP-subjected mice and LPS-challenged BMDMs. Furthermore, the production of iNOS and NO was also decreased after fisetin treatment. These effects of fisetin on macrophages indicate that fisetin is a promising agent to suppress macrophage activation and protect against sepsis.

Inhibition of TLR4 and TLR4-mediated signaling has been regarded as a method for controlling sepsis [37]. TAK1 and TAB1 play important roles in the TLR4-mediated inflammatory response [38]. The formation of the TAK1-TAB1 complex is necessary for autophosphorylation-induced TAK1 activation $[38,39]$. Therefore, inhibiting the interaction between TAK1 and TAB1 is regarded as a potential approach to modulate activated TLR4 signaling and suppress subsequent inflammatory responses. Several studies have applied this theory in controlling inflammatory diseases and screening new compounds to mitigate inflammation. For example, both stercurensin and curcumin metabolism inhibit NF-KB-dependent inflammation by attenuating the formation of the TAK1-TAB1 complex $[40,41]$. Although the suppressive effect of fisetin in TNF-a-induced TAK1 activation has been previously reported, no direct evidence has explained its role in the interaction between TAK1 and TAB1 [42]. Our findings showed that fisetin reduced the interaction between TAK1 and TAB1 and suppressed LPS-induced TAK1 activation, suggesting an inhibitory effect of the fisetin on the TLR4-mediated signaling pathway. The inhibitory potential of fisetin on the TAK1-TAB1 complex needs to be explored further through research.

In addition to NF-KB signaling, MAPK also serves as a significant regulator in LPS-induced inflammation. Activation of MAPK is closely associated with the increased production of various chemokines and cytokines, which promotes the inflammatory response [43]. In addition, as a downstream effector of p38 MAPK, MK2 plays a critical role in determining cell fate in the inflammatory response to infection [44]. MK2 deficiency suppressed the release of inflammatory mediators in macrophages and protected mice from sepsis-induced ALI. In addition, pharmacological inhibition of p38 MAPK alleviated inflammation in ALI $[45,46]$. An increasing number of studies of compounds show modulation of inflammatory processes via the MAPK signaling pathway. For instance, indirubin suppressed the MAPK signaling pathway in LPS-induced inflammation, in which ERK and p38 MAPK were attenuated [47]. Our study suggests that the antiinflammatory effects of fisetin result from the inhibition of the p38 MAPK/MK2 signaling pathway.

Recent studies have revealed the diverse applications of fisetin. The potential role of fisetin in gastric cancer has been demonstrated in mitochondria-driven cell death [25]. Fisetin protected rats from aging-induced oxidative stress and neurodegeneration by activating the PI3K-Akt signaling pathway $[48,49]$. In addition to performing the anti-inflammatory activity of fisetin in vivo and in vitro, our study confirms the protective role of fisetin in sepsisinduced multiple organ failure, which results from inhibiting the binding of TAK1 and TAB1.

In conclusion, our study demonstrated that fisetin prevented severe injuries in multiple organs by suppressing the inflammatory response in a murine CLP model. We also found that fisetin inhibited the phosphorylation of p38 MAPK/MK2 by suppressing the interaction between TAK1 and TAB1. These findings confirm 


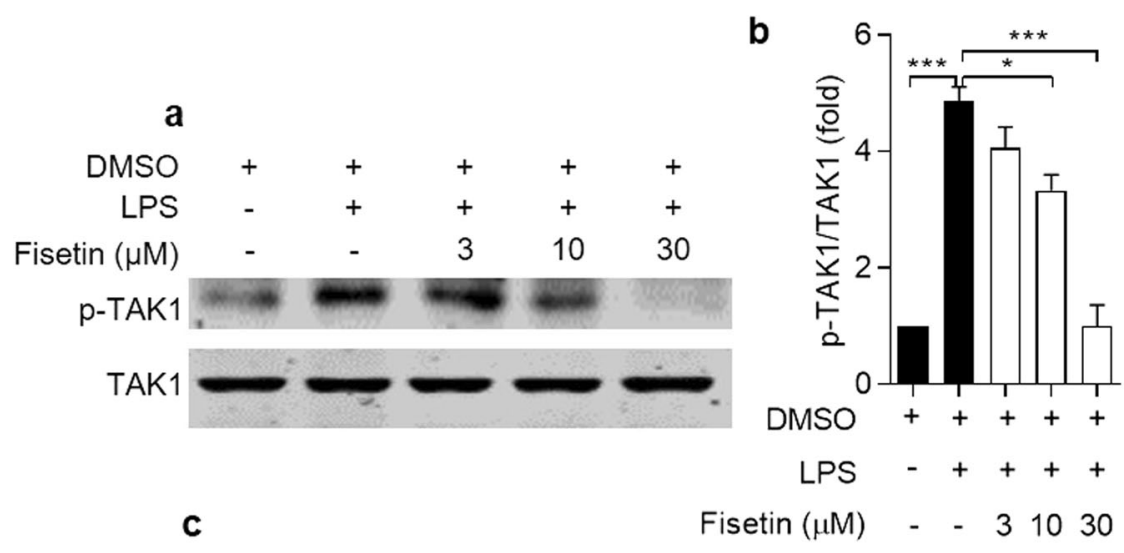

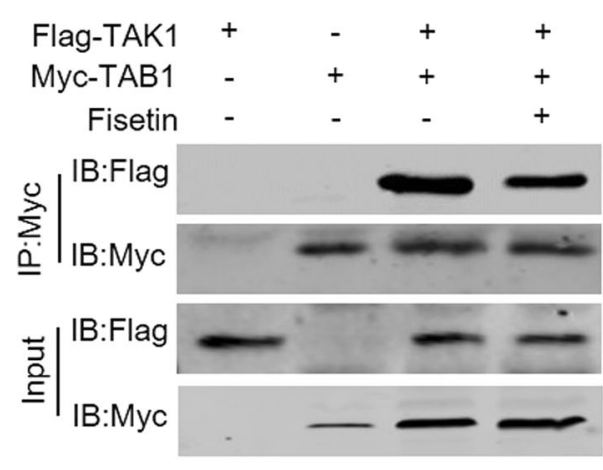

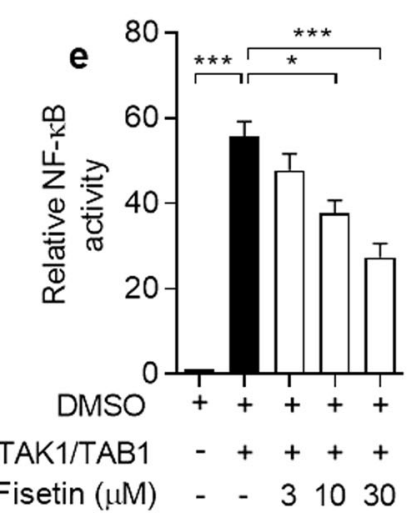

d

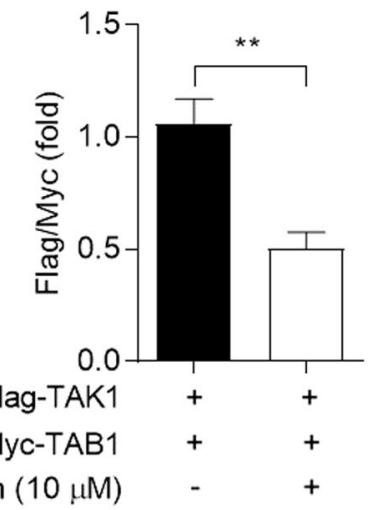

Fig. 7 Fisetin suppresses $\mathbf{p} 38$ and NF-KB activity by targeting TAK1/TAB1. a The protein expression levels of p-TAK1 and TAK1 were evaluated by Western blotting. b Statistical analysis of p-TAK1, as shown in the histograms. c Flag-TAK1 and Myc-TAB1 were cotransfected into HEK293T cells. The effect of fisetin on protein interactions was determined by immunoprecipitation assay. Total cell lysates were used as the input control. d Statistical analysis of (c), as shown in the histograms. e Eighteen hours after transfection with TAK1/TAB1, fisetin was added at different concentrations, and the relative NF- $\mathrm{KB}$ activity in the HEK293T cells was determined $6 \mathrm{~h}$ later using a luciferase reporter assay. The results are expressed as the means \pm SEM of three independent experiments $\left({ }^{*} P<0.05\right.$, ${ }^{* *} P<0.01$, and $\left.{ }^{* * *} P<0.001\right)$.

the anti-sepsis and anti-inflammation role for fisetin, suggesting it a potential reagent in sepsis management.

\section{ACKNOWLEDGEMENTS}

This work was supported by the Natural Science Research Project of Education Office of Anhui Province (KJ2019A0300); The National Key Clinical Specialty Construction Project of Pulmonary Critical Care Medicine (2012-649); The Key Research and Development Program of Anhui Province (1804h08020287); and National Natural Science Foundation of China (81673791, 81973329, 81773741, and 81573438).

\section{AUTHOR CONTRIBUTIONS}

HFZ and FQ designed the study. HFZ and HBZ performed the experiments and drafted the manuscript; XPW and YLG participated in data analysis; WDC was involved in discussion of the experiments. All authors read and approved the final manuscript.

\section{ADDITIONAL INFORMATION}

Competing interests: The authors declare no competing interests.

\section{REFERENCES}

1. Vincent JL, Opal SM, Marshall JC, Tracey KJ. Sepsis definitions: time for change. Lancet. 2013;381:774-5.

2. Hotchkiss RS, Monneret G, Payen D. Sepsis-induced immunosuppression: from cellular dysfunctions to immunotherapy. Nat Rev Immunol. 2013;13: 862-74.

3. Martin GS, Mannino DM, Moss M. The effect of age on the development and outcome of adult sepsis. Crit Care Med. 2006;34:15-21. 
4. Suffredini AF, Munford RS. Novel therapies for septic shock over the past 4 decades. JAMA. 2011;306:194-9.

5. Kaukonen KM, Bailey M, Suzuki S, Pilcher D, Bellomo R. Mortality related to severe sepsis and septic shock among critically ill patients in Australia and New Zealand, 2000-2012. JAMA. 2014;311:1308-16.

6. Vincent JL, Marshall JC, Namendys-Silva SA, François B, Martin-Loeches I, Lipman $J$, et al. Assessment of the worldwide burden of critical illness: the intensive care over nations (ICON) audit. Lancet. Respir Med. 2014;2:380-6.

7. Cohen J. The immunopathogenesis of sepsis. Nature. 2002;420:885-91.

8. Cohen J, Vincent JL, Adhikari NK, Machado FR, Angus DC, Calandra T, et al. Sepsis: a roadmap for future research. Lancet Infect Dis. 2015;15:581-614.

9. Andersson U, Tracey KJ. HMGB1 is a therapeutic target for sterile inflammation and infection. Annu Rev Immunol. 2011;29:139-62.

10. Cruz DN, Perazella MA, Bellomo R, de Cal M, Polanco N, Corradi V, et al. Effectiveness of polymyxin B-immobilized fiber column in sepsis: a systematic review. Crit Care. 2007;11:R47.

11. Kumar V. Targeting macrophage immunometabolism: dawn in the darkness of sepsis. Int Immunopharmacol. 2018;58:173-85.

12. Hamidzadeh K, Christensen SM, Dalby E, Chandrasekaran P, Mosser DM. Macrophages and the recovery from acute and chronic inflammation. Annu Rev Physiol. 2017;79:567-92.

13. Vergadi $E$, leronymaki $E$, Lyroni $K$, Vaporidi $K$, Tsatsanis C. Akt signaling pathway in macrophage activation and M1/M2 polarization. J Immunol. 2017;198:1006-14.

14. Chow A, Huggins M, Ahmed J, Hashimoto D, Lucas D, Kunisaki Y, et al. CD169 ${ }^{+}$ macrophages provide a niche promoting erythropoiesis under homeostasis and stress. Nat Med. 2013;19:429-36.

15. Kawai T, Akira S. The role of pattern-recognition receptors in innate immunity: update on Toll-like receptors. Nat Immunol. 2010;11:373-84.

16. van Lookeren Campagne M, Wiesmann C, Brown EJ. Macrophage complement receptors and pathogen clearance. Cell Microbiol. 2007;9:2095-102.

17. Elieh Ali Komi D, Sharma L, Dela Cruz CS. Chitin and its Effects on inflammatory and immune responses. Clin Rev Allergy Immunol. 2018;54:213-23.

18. He HQ, Wu YX, Nie YJ, Wang J, Ge M, Qian F. LYRM03, an ubenimex derivative, attenuates LPS-induced acute lung injury in mice by suppressing the TLR4 signaling pathway. Acta Pharmacol Sin. 2017;38:342-50.

19. Kuzmich NN, Sivak KV, Chubarev VN, Porozov YB, Savateeva-Lyubimova TN, Peri F. TLR4 signaling pathway modulators as potential therapeutics in inflammation and sepsis. Vaccines (Basel). 2017;5:E34. pii

20. Lu YC, Yeh WC, Ohashi PS. LPS/TLR4 signal transduction pathway. Cytokine. 2008;42:145-51.

21. Shinohara $H$, Yasuda $T$, Kurosaki T. TAK1 adaptor proteins, TAB2 and TAB3, link the signalosome to B-cell receptor-induced IKK activation. FEBS Lett. 2016;590:3264-9.

22. Yoo H, Ku SK, Han MS, Kim KM, Bae JS. Anti-septic effects of fisetin in vitro and in vivo. Inflammation. 2014;37:1560-74.

23. Wang $T H$, Wang SY, Wang $X D$, Jiang $H Q$, Yang $Y Q$, Wang $Y$, et al. Fisetin exerts antioxidant and neuroprotective effects in multiple mutant hSOD1 models of amyotrophic lateral sclerosis by activating ERK. Neuroscience. 2018;379:152-66.

24. Zhang $H$, Zheng W, Feng $X$, Yang F, Qin $H$, Wu S, et al. Nrf2(-)ARE signaling acts as master pathway for the cellular antioxidant activity of fisetin. Molecules. 2019;24: E708. pii

25. Sabarwal A, Agarwal R, Singh RP. Fisetin inhibits cellular proliferation and induces mitochondria-dependent apoptosis in human gastric cancer cells. Mol Carcinog. 2017;56:499-514.

26. Peng $\mathrm{HL}$, Huang $\mathrm{WC}$, Cheng $\mathrm{SC}$, Liou CJ. Fisetin inhibits the generation of inflammatory mediators in interleukin-1 beta-induced human lung epithelial cells by suppressing the NF-kappaB and ERK1/2 pathways. Int Immunopharmacol. 2018;60:202-10.

27. Khakpour S, Wilhelmsen K, Hellman J. Vascular endothelial cell Toll-like receptor pathways in sepsis. Innate Immun. 2015;21:827-46.

28. Ward PA, Gao H. Sepsis, complement and the dysregulated inflammatory response. J Cell Mol Med. 2009;13:4154-60.

29. Pfalzgraff A, Heinbockel L, Su Q, Brandenburg K, Weindl G, Brandenburg G. Synthetic anti-endotoxin peptides inhibit cytoplasmic LPS-mediated responses. Biochem Pharmacol. 2017;140:64-72.
30. Ge $Y, X u X$, Liang $Q, X u ~ Y$, Huang M. Alpha-Mangostin suppresses NLRP3 inflammasome activation via promoting autophagy in LPS-stimulated murine macrophages and protects against CLP-induced sepsis in mice. Inflamm Res. 2019;68:471-9.

31. Durairaj H, Steury MD, Parameswaran N. Paroxetine differentially modulates LPSinduced TNFalpha and IL-6 production in mouse macrophages. Int Immunopharmacol. 2015;25:485-92.

32. Lukacs NW, Strieter RM, Chensue SW, Widmer M, Kunkel SL. TNF-alpha mediates recruitment of neutrophils and eosinophils during airway inflammation. J Immunol. 1995;154:5411-7.

33. Linkermann A, Stockwell BR, Krautwald S, Anders HJ. Regulated cell death and inflammation: an auto-amplification loop causes organ failure. Nat Rev Immunol. 2014;14:759-67.

34. Newham P, Ross D, Ceuppens P, Das S, Yates JW, Betts C, et al. Determination of the safety and efficacy of therapeutic neutralization of tumor necrosis factoralpha (TNF-alpha) using AZD9773, an anti-TNF-alpha immune Fab, in murine CLP sepsis. Inflamm Res. 2014;63:149-60.

35. Liu Y, Perumalsamy H, Kang CH, Kim SH, Wang JS, Koh SC, et al. Intracellular synthesis of gold nanoparticles by Gluconacetobacter liquefaciens for delivery of peptide CopA3 and ginsenoside and anti-inflammatory effect on lipopolysaccharide-activated macrophages. Artif Cells Nanomed Biotechnol. 2020:48:777-88.

36. Heemskerk S, Masereeuw R, Russel FG, Pickkers P. Selective iNOS inhibition for the treatment of sepsis-induced acute kidney injury. Nat Rev Nephrol. 2009;5:629-40.

37. Opal SM, Laterre PF, Francois B, LaRosa SP, Angus DC, Mira JP, et al. Effect of eritoran, an antagonist of MD2-TLR4, on mortality in patients with severe sepsis: the ACCESS randomized trial. JAMA. 2013;309:1154-62.

38. Chen IT, Hsu PH, Hsu WC, Chen NJ, Tseng PH. Polyubiquitination of transforming growth factor beta-activated kinase 1 (TAK1) at Lysine 562 residue regulates TLR4-mediated JNK and p38 MAPK Activation. Sci Rep. 2015;5:12300.

39. Hirata $Y$, Takahashi M, Morishita T, Noguchi T, Matsuzawa A. Post-translational modifications of the TAK1-TAB complex. Int J Mol Sci. 2017;18:E205. pii

40. Kim YJ, Kim HC, Ko H, Amor EC, Lee JW, Yang HO, et al. Stercurensin inhibits nuclear factor-kappaB-dependent inflammatory signals through attenuation of TAK1-TAB1 complex formation. J Cell Biochem. 2011;112:548-58.

41. Zhang ZB, Luo DD, Xie JH, Xian YF, Lai ZQ, Liu YH, et al. Curcumin's metabolites, tetrahydrocurcumin and octahydrocurcumin, possess superior anti-inflammatory effects in vivo through suppression of TAK1-NF-kappaB pathway. Front Pharmacol. 2018;9:1181. https://doi.org/10.3389/fphar.2018.01181.

42. Sung B, Pandey MK, Aggarwal BB. Fisetin, an inhibitor of cyclin-dependent kinase 6, down-regulates nuclear factor-kappaB-regulated cell proliferation, antiapoptotic and metastatic gene products through the suppression of TAK-1 and receptor-interacting protein-regulated IkappaBalpha kinase activation. Mol Pharmacol. 2007;71:1703-14.

43. Oberst A. MK2 balances inflammation and cell death. Nat Cell Biol. 2017;19:1150-2.

44. Menon MB, Gropengießer J, Fischer J, Novikova L, Deuretzbacher A, Lafera J, et al. p38(MAPK)/MK2-dependent phosphorylation controls cytotoxic RIPK1 signalling in inflammation and infection. Nat Cell Biol. 2017;19:1248-59.

45. Wu Y, He H, Ding Y, Liu S, Zhang D, Wang J, et al. MK2 mediates macrophage activation and acute lung injury by regulating let-7e miRNA. Am J Physiol-Lung C. 2018:315:L371-L81.

46. Chen YT, Du Y, Zhao B, Gan LX, Yu KK, Sun L, et al. Costunolide alleviates HKSAinduced acute lung injury via inhibition of macrophage activation. Acta Pharmacol Sin. 2019;40:1040-8

47. Lai JL, Liu YH, Liu C, Qi MP, Liu RN, Zhu XF, et al. Indirubin inhibits LPS-induced inflammation via TLR4 abrogation mediated by the NF-KB and MAPK signaling pathways. Inflammation. 2017;40:1-12.

48. Singh S, Singh AK, Garg G, Rizvi SI. Fisetin as a caloric restriction mimetic protects rat brain against aging induced oxidative stress, apoptosis and neurodegeneration. Life Sci. 2018;193:171-9.

49. Watanabe R, Kurose T, Morishige $Y$, Fujimori K. Protective effects of fisetin against 6-OHDA-induced apoptosis by activation of PI3K-Akt signaling in human neuroblastoma SH-SY5Y cells. Neurochem Res. 2018;43:488-99. 